\title{
Verification of modulus and fatigue cracking models for hot-mix asphalt with asbuton in laboratory scale
}

\author{
Juan Nugraha ${ }^{1, *}$, Djunaedi Kosasih ${ }^{2}$, and Harmein Rahman $^{2}$ \\ ${ }^{1}$ Highway Engineering and Development Master Study Program, Faculty of Civil and Environmental Engineering, Institut Teknologi \\ Bandung, Bandung, Indonesia \\ ${ }^{2}$ Transportation Engineering Research Group, Faculty of Civil and Environmental Engineering, Institut Teknologi Bandung, Bandung, \\ Indonesia
}

\begin{abstract}
Design and analysis of flexible pavement structure using mechanistic approaches require, among other input data, the dynamic modulus of asphalt layer $\left(\mathrm{E}^{*}\right)$ and it's resistance to fatigue cracking $\left(\mathrm{N}_{\mathrm{f}}\right)$. These material characteristics can be obtained from both laboratory test results and calibrated against field conditions and from mathematical models, such as the Asphalt Institute's. The two values are to be compared for assessing the applicability of the models for mixes using petroleum bitumen (pen 60/70) and using modified asphalt (with $8 \%$ asbuton). The laboratory tests were conducted using Asphalt Mixture Performance Test (AMPT) and Four Point Loading equipment. It was found that the resulting curves are consistent with the Asphalt Institute's model for both types of mixes. Meanwhile, fatigue life model curves show a similar trend to the Asphalt Institute's model on the conservative side. This is explainable the laboratory model needs to be calibrated for variations of wheel track and loading time occuring on site.
\end{abstract}

\section{Introduction}

Design and analysis of flexible pavement structure using mechanistic approaches require the dynamic modulus of asphalt layer $\left(\mathrm{E}^{*}\right)$ and it's resistance to fatigue crack (fatigue life) values.

The dynamic modulus is a stress-strain relationship for a linear viscoelastic material that accepts a sinusoidal load of a certain frequency (N. Suaryana, 2014).

Fatigue cracking is a phenomenon of cracking due to recurrent loads that occur due to repetition of strain still under the strength of the material (E.J Yoder and M.W Witczak, 1975).

The values mentioned above, in addition can be obtained from the test results in the laboratory, can also be obtained from mathematical model.

Models of asphalt mixed modulus have been widely developed, among others modulus models by Witczak (2006), Asphalt Institute (1982), Bonnaure et.al (1977) and Nottingham (Brown, et al., 1984). In this research, the dynamic modulus model used is the Asphalt Institute (AI) model.

The fatigue life $\left(\mathrm{N}_{\mathrm{f}}\right)$ models is derived based on different laboratory testing methods:

a. Constant Stress : Asphalt Institute (AI), Shell ;

b. Constant Strain : Shell, Austroad, Nottingham, SHRPBerkeley ;

c. Full Scale: Transport and Road Research Laboratory (TRRL).
This research will use the method (a) constant stress and $\mathrm{N}_{\mathrm{f}}$ model by Asphalt Institute. This model is chosen because the parameters in the calculation is complete enough that there is a function of strain $(\varepsilon)$, dynamic modulus of asphalt layers $\left(\mathrm{E}^{*}\right)$ as well as mixed volumetric parameters.

Statistical analysis was performed to verify both models to see consistency between test data and model calculations. Through verification of modulus and fatigue cracking models, the dynamic modulus of asphalt layer $\left(\mathrm{E}^{*}\right)$ and fatigue life $\left(\mathrm{N}_{\mathrm{f}}\right)$ values can be obtained with models, without having to carry out expensive and complicated laboratory testing. So that can optimize time and cost when doing the design and analysis of flexible pavement structure using a mechanistic approaches.

\section{Theoritical background}

\subsection{Dynamic modulus $\left(E^{*}\right)$ model for asphalt}

The Asphalt Institute (1982) states that the dynamic modulus of hot mix asphalt (HMA) is a function of the percentage of aggregates that passes the filter no. 200, loading frequency, air void, asphalt viscosity, temperature and asphalt volume percentage with the following equation:

$$
\left|E^{*}\right|=100,000 \times 10^{\beta_{1}}
$$

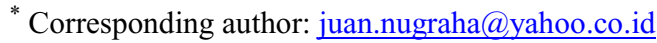


With:

$$
\begin{aligned}
& \beta_{1}=\beta_{3}+0.000005 \beta_{2}-0.00189 \beta_{2} f^{-1.1} \\
& \beta_{2}=\beta_{4}{ }^{0.5} T^{\beta_{5}} \\
& \beta_{3}=\quad 0.553833+0.02882\left(P_{200} f^{-0.1703}\right)-
\end{aligned}
$$

$0.003476 V_{a}+0.070377 \lambda+0.931757 f^{-0.002774}$

$$
\begin{aligned}
& \beta_{4}=0.483 V_{b} \\
& \beta_{5}=1.3+0.49825 \log f \\
& \lambda=29,508.2\left(P_{77^{0} F}\right)^{-2.1939}
\end{aligned}
$$

Where,

$\beta 1, \beta 2, \beta 3, \beta 4, \beta 5=$ temporary constants

$f$ = loading frequency, $(\mathrm{Hz})$

$\mathrm{P}_{200}=$ percent of aggregates passed the filter no. $200,(\%)$

$\mathrm{Vb}=$ asphalt volume, (\%)

$\lambda=$ viscosity of bitumen on $70^{0} \mathrm{~F},\left(10^{6}\right.$ poises $)$

$\mathrm{T}=$ temperature of asphalt mixture, $\left({ }^{0} \mathrm{~F}\right)$

\subsection{Fatigue model}

Fatigue life $\left(\mathrm{N}_{\mathrm{f}}\right)$ of HMA can be predicted by performing calculations through a mathematical model. The fatigue model reviewed here is only the Asphalt Institute model with constant stress testing using the Four Point Loading which is calibrated by Witczak and El-Basyouny for American Mechanistic-Empirical Pavement Design Guide (MEPDG) are as follows (J. Yu and G. Zou, 2013):

$$
N_{f}=18.4 \times 0.00432 C \varepsilon_{t}{ }^{-3.291}\left|E^{*}\right|^{-0.854}
$$

Where $\mathrm{C}$ is a correction factor, $\mathrm{C}=10^{\mathrm{M}}$

$$
M=4.84\left[\frac{V b}{V a+V b}-0.69\right]
$$

Where :

$\mathrm{N}_{\mathrm{f}}=$ fatigue life to failure, cycle

$\mathrm{C}=$ correction factor $=10^{\mathrm{M}}$

$=$ function of asphalt volume and volume of air void

$\varepsilon_{\mathrm{t}} \quad=$ tensile strain, $\mu \varepsilon$

$\left|E^{*}\right|=$ dynamic modulus complex of asphalt mixture, Psi

$\mathrm{V}_{\mathrm{b}}=$ percentage of asphalt volume (bitumen) in the mixture, $\%$

$\mathrm{V}_{\mathrm{a}}=$ volume of air void in mixture, $\%$

\section{Test result and analysis}

\subsection{Dynamic modulus}

The dynamic asphalt mixed modulus testing uses AMPT and making of the sample using a gyratory compactor. Dynamic modulus testing was performed with sinusoidal loading at $20^{\circ} \mathrm{C}, 35^{\circ} \mathrm{C}, 50^{\circ} \mathrm{C}$, for each temperature tested at a frequency of $0.01 \mathrm{~Hz} ; 0.1 \mathrm{~Hz} ; 1 \mathrm{~Hz} ; 10 \mathrm{~Hz}$.

The summary of HMA with $8 \%$ asbuton, dynamic modulus test results is presented in Table 1, whereas the results for HMA without asbuton show the same behavior. Then for mathematical calculations the modulus model can be seen in Table 2 .

To compare the results of the dynamic modulus testing of the AMPT test with the modulus of the Four Point Loading test and the modulus model according to the Asphalt Institute, the first step is to mathematically calculate the model based on equations 1 to 7 .

Furthermore, to obtain the relationship between the Four Point Loading modulus test results with the AMPT test results need to adjust the loading frequency, because the Four Point Loading test is done at the frequency of $8.06 \mathrm{~Hz}$, while the AMPT test at the frequency of 0.01 ; $0.1 ; 1 ; 10 \mathrm{~Hz}$. The step taken is to perform a linear interpolation between the AMPT test results and the Four Point Loading test results.

All modulus values are then plotted into the graph of the temperature relation $\left({ }^{\circ} \mathrm{C}\right)$ with the modulus shown in Figure 2 and 3. To see the consistency between these values, a statistical test is performed, complete verification steps are presented in Figure 1. As for the other mixtures showing the same behavior.

In Figure 2 dan 3, shows of HMA without and with $8 \%$ asbuton, at temperature and all frequencies $(0.01 \mathrm{~Hz}$; $0.1 \mathrm{~Hz} ; 1 \mathrm{~Hz} ; 10 \mathrm{~Hz}$ ) for the three variations of asphalt content $(5 \% ; 6 \% ; 7 \%)$, the value of the test modulus is mostly above the model.

When observed on the graphs of the dynamic modulus, at a frequency of $0.01 ; 0.1 ; 10 \mathrm{~Hz}$, AI model line and the AMPT test line look consistent, where in the 0.01 $\mathrm{Hz}$ and $0.1 \mathrm{~Hz}$ frequencies the lines are both concave, as does the AMPT test line. Then for the $10 \mathrm{~Hz}$ frequency the AI convex model line, same as the AMPT test line. But somewhat distorted occurs in the frequency of $1 \mathrm{~Hz}$ (black line), where the model line tends to convex, while for the AMPT test line tends to be concave. This needs further research to find the cause of the phenomenon.

The dynamic modulus relationship of the AMPT test results and the AI model shown in Figure 4, most of the modulus values of the test results are greater than the model. This, if seen from its influence on flexible pavement design, that the model is safer to use in planning, because it produces a lower modulus, which in fact in the asphalt layers is built has a greater modulus value, indicated by the results of AMPT testing.

Table 1. AMPT test results for HMA with $8 \%$ asbuton

\begin{tabular}{|c|c|c|c|c|c|c|c|}
\hline \multicolumn{2}{|c|}{ Condition } & \multicolumn{2}{c|}{ Asphalt Content 5\% } & \multicolumn{2}{c|}{ Asphalt Content 6\% } & \multicolumn{2}{c|}{ Asphalt Content 7\% } \\
\hline Temperature & Frequency & $\begin{array}{c}\text { Dynamic } \\
\text { Modulus }\end{array}$ & Phase Angle & $\begin{array}{c}\text { Dynamic } \\
\text { Modulus }\end{array}$ & Phase Angle & $\begin{array}{c}\text { Dynamic } \\
\text { Modulus }\end{array}$ & Phase Angle \\
\hline \hline${ }^{\circ} \mathrm{C}$ & $\mathrm{Hz}$ & $\mathrm{MPa}$ & $\left({ }^{\circ}\right)$ & $\mathrm{Mpa}$ & $\left.{ }^{\circ}\right)$ & $\mathrm{Mpa}$ & $\left({ }^{\circ}\right)$ \\
\hline 20 & 0.01 & $1,907.433$ & 36.58 & $1,034.300$ & 35.13 & 828.935 & 34.46 \\
\hline 20 & 0.1 & $5,141.885$ & 32.11 & $3,036.143$ & 35.52 & $2,312.302$ & 36.70 \\
\hline 20 & 1 & $10,063.510$ & 22.10 & $6,816.994$ & 27.24 & $5,386.530$ & 29.19 \\
\hline \hline 20 & 10 & $16,153.230$ & 14.24 & $11,555.780$ & 18.65 & $9,702.566$ & 19.54 \\
\hline 35 & 0.01 & 523.316 & 24.47 & 292.589 & 19.44 & 305.760 & 19.62 \\
\hline 35 & 0.1 & $1,294.000$ & 35.30 & 509.264 & 32.18 & 703.296 & 34.67 \\
\hline 35 & 1 & $3,444.867$ & 35.09 & $1,400.384$ & 41.82 & $2,049.840$ & 38.95 \\
\hline \hline
\end{tabular}




\begin{tabular}{|c|c|c|c|c|c|c|c|}
\hline \multicolumn{2}{|c|}{ Condition } & \multicolumn{2}{c|}{ Asphalt Content 5\% } & \multicolumn{2}{c|}{ Asphalt Content 6\% } & \multicolumn{2}{c|}{ Asphalt Content 7\% } \\
\hline Temperature & Frequency & $\begin{array}{c}\text { Dynamic } \\
\text { Modulus }\end{array}$ & Phase Angle & $\begin{array}{c}\text { Dynamic } \\
\text { Modulus }\end{array}$ & Phase Angle & $\begin{array}{c}\text { Dynamic } \\
\text { Modulus }\end{array}$ & Phase Angle \\
\hline \hline${ }^{\circ} \mathrm{C}$ & $\mathrm{Hz}$ & $\mathrm{MPa}$ & $\left({ }^{\circ}\right)$ & $\mathrm{Mpa}$ & $\left.{ }^{\circ}\right)$ & $\mathrm{Mpa}$ & $\left(^{\circ}\right)$ \\
\hline 50 & 0.01 & 281.792 & 11.29 & 207.553 & 11.00 & 194.003 & 8.78 \\
\hline 50 & 0.1 & 352.448 & 18.60 & 279.826 & 21.95 & 234.765 & 15.94 \\
\hline 50 & 1 & 629.325 & 30.98 & 563.473 & 37.91 & 396.026 & 29.23 \\
\hline 50 & 10 & $1,698.911$ & 39.36 & $1,695.248$ & 42.03 & $1,102.264$ & 39.67 \\
\hline
\end{tabular}

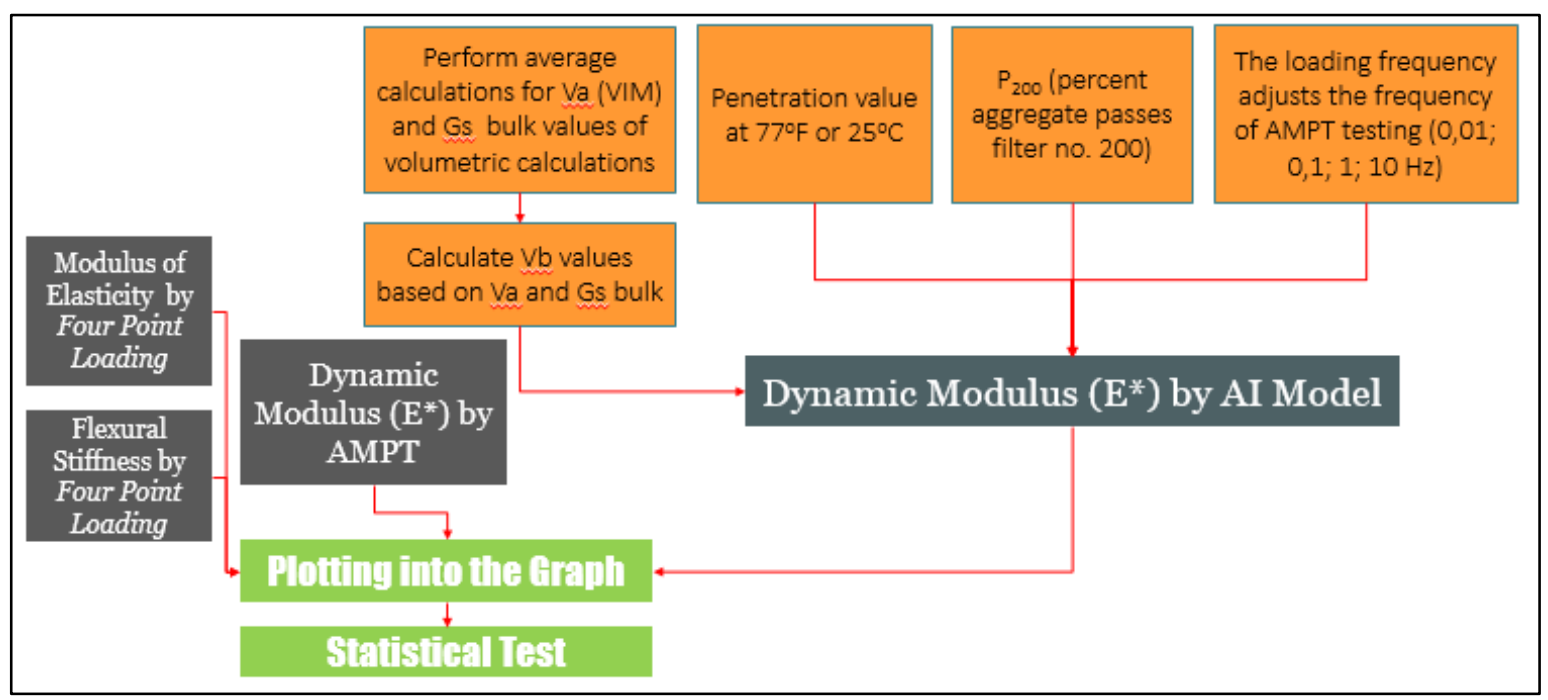

Fig. 1. The steps to verify the AMPT test results on the HMA modulus model

Tabel 2. Results of calculation of dynamic modulus AI model with the frequency according to the modulus testing of AMPT for HMA with $8 \%$ asbuton

\begin{tabular}{|c|c|c|c|c|c|c|c|c|c|c|c|c|c|c|c|c|c|c|c|c|}
\hline No. & Bl & B2 & B3 & B4 & B5 & $\mathrm{f}(\mathrm{Hz})$ & $\log f$ & P200 & $\begin{array}{c}\mathrm{Vv}(\%)= \\
\mathrm{VIM}\end{array}$ & $\begin{array}{l}\text { VFB } \\
(\%)\end{array}$ & $\begin{array}{l}\text { VMA } \\
(\%)\end{array}$ & $\begin{array}{l}\mathrm{Vb}(\%) \\
=\mathrm{Vfbx} \\
\mathrm{VMA}\end{array}$ & $\lambda$ & $\begin{array}{c}\mathrm{P} 77^{\circ} \mathrm{F} \\
(\operatorname{Pen} 25 \\
\left.{ }^{\circ} \mathrm{C}\right)\end{array}$ & $E$ (psi) & $\mathrm{E}(\mathrm{MPa})$ & $\begin{array}{c}\mathrm{T} \\
(\mathrm{C})\end{array}$ & $\begin{array}{c}\mathrm{T} \\
(\mathrm{F})\end{array}$ & $\begin{array}{l}\text { AMPT } \\
\text { Test }\end{array}$ & \\
\hline \multirow{3}{*}{ 5.1a } & $\mid 0.0467$ & 7.5699 & 3142 & 4.4244 & 0.3035 & 0.01 & -2.00000 & 6.5 & 8.649 & 51.444 & 17.806 & 9.160 & 8.4083 & 41.3 & $111,356.92$ & 767.78 & 20 & 68 & $1,907.43$ & \multirow{3}{*}{$\begin{array}{c}\text { Pen } 60 / 70+\text { Asb } \\
8 \%(5 \%)\end{array}$} \\
\hline & -0.1955 & 8.3785 & 2.3142 & 4.4244 & 0.3035 & 0.01 & -2.00000 & 6.5 & 8.649 & 51.444 & 17.806 & 9.160 & 8.4083 & 41.3 & $63,757.03$ & 439.59 & 35 & 95 & 523.32 & \\
\hline & $|-0.3934|$ & 9.0393 & 2.3142 & 4.4244 & 0.3035 & 0.01 & -2.00000 & 6.5 & 8.649 & 51.444 & 17.806 & 9.160 & 8.4083 & 41.3 & $40,418.17$ & 278.68 & 50 & 122 & 281.79 & \\
\hline \multirow{3}{*}{$5.2 \mathrm{a}$} & 0.6524 & 61.5061 & 2.1155 & 4.3592 & 0.8018 & 0.1 & -1.00000 & 6.5 & 8.649 & 51.444 & 17.544 & & 8.4083 & 41.3 & $449,146.29$ & $3,096.77$ & 20 & 68 & $5,141.89$ & \multirow{3}{*}{$\begin{array}{c}\text { Pen } 60 / 70+\text { Asb } \\
8 \%(5 \%)\end{array}$} \\
\hline & 0.2025 & 80.4163 & 2.1155 & 4.3592 & 0.8018 & 0.1 & -1.00000 & 6.5 & 8.649 & 51.444 & 17.544 & 9.025 & 8.4083 & 41.3 & $159,418.60$ & $1,099.16$ & 35 & 95 & $1,294.00$ & \\
\hline & -0.2223 & 98.2750 & 2.1155 & 4.3592 & 0.8018 & 0.1 & -1.00000 & 6.5 & 8.649 & 51.444 & 17.544 & 9.025 & 8.4083 & 41. & $59,938.13$ & 413.26 & 50 & 122 & 352.45 & \\
\hline \multirow{3}{*}{$5.3 \mathrm{a}$} & 1.0151 & 503.4609 & 1.9641 & 4.3592 & 1.3000 & 1 & 0.00000 & 6.5 & 8.649 & 51.444 & 17.544 & 9.025 & 8.4083 & 41. & $1,035,347.27$ & $7,138.51$ & 20 & 68 & $10,063.51$ & \multirow{3}{*}{$\begin{array}{c}\text { Pen } 60 / 70+\text { Asb } \\
8 \%(5 \%)\end{array}$} \\
\hline & \begin{tabular}{|l|}
0.4984 \\
\end{tabular} & 777.5796 & 1.9641 & 4.3592 & 1.3000 & 1 & 0.00000 & 6.5 & 8.649 & 51.444 & 17.544 & 9.025 & 8.4083 & 41.3 & $315,044.79$ & $2,172.17$ & 35 & 95 & $3,444.87$ & \\
\hline & -0.0649 & 1076.3957 & 1.9641 & 4.3592 & 1.3000 & 1 & 0.00000 & 6.5 & 8.649 & 51.444 & 17.544 & 9.025 & 8.4083 & 41. & $86,119.99$ & 593.78 & 50 & 122 & 629.33 & \\
\hline \multirow{3}{*}{$5.4 \mathrm{a}$} & 1.2476 & 4121.1017 & 1.8457 & 4.3592 & 1.7983 & 10 & 1.00000 & 6.5 & 8.649 & 51.444 & 17.544 & 9.025 & 8.4083 & 41.3 & $1,768,409.27$ & $12,192.83$ & 20 & 68 & $\begin{array}{l}6,153.23 \\
\end{array}$ & \multirow{3}{*}{$\begin{array}{c}\text { Pen } 60 / 70+A s b \\
8 \%(5 \%)\end{array}$} \\
\hline & \begin{tabular}{|l|l|}
0.7545 \\
\end{tabular} & 7518.7502 & 1.8457 & 4.3592 & 1.7983 & 10 & 1.00000 & 6.5 & 8.649 & 51.444 & 17.544 & 9.025 & 8.4083 & 41. & & $3,917.51$ & 35 & 95 & $7,397.91$ & \\
\hline & \begin{tabular}{|l|}
0.1347 \\
\end{tabular} & 11789.6456 & 1.8457 & 4.3592 & 1.7983 & 10 & 1.00000 & 6.5 & 8.649 & 51.444 & 17.544 & 9.025 & 8.4083 & 41. & 52.18 & 940.12 & 50 & 122 & $1,698.91$ & \\
\hline \multirow{3}{*}{ 6.la } & -0.1352 & 8.4793 & 2.4047 & 5.5512 & 0.3035 & 0.01 & -2.00000 & 6.5 & 6.046 & 65.545 & 17.535 & 11.493 & 8.4083 & 41 & 244.76 & 505.01 & 20 & 68 & $1,034.30$ & \multirow{3}{*}{$\begin{array}{c}\text { Pen } 60 / 70+A s b \\
8 \%(6 \%)\end{array}$} \\
\hline & -0.4065 & 9.3850 & 2.4047 & 5.5512 & 0.3035 & 0.01 & -2.00000 & 6.5 & & 65.545 & 17.535 & 11.493 & 8.4083 & & 218.70 & 270.41 & 35 & 95 & 292.59 & \\
\hline & -0.6282 & 10.1252 & 2.4047 & 5.5512 & 0.3035 & 0.01 & -2.00000 & 6.5 & 6.046 & 65.545 & 17.535 & 11.493 & 8.4083 & & $23,537.58$ & 162.29 & 50 & 122 & 207.55 & \\
\hline \multirow{3}{*}{$6.2 \mathrm{a}$} & 0.5549 & 69.4079 & 2.2060 & 5.5512 & 0.8018 & 0.1 & -1.00000 & 6.5 & 6.046 & 65.545 & 17.535 & 11.493 & 8.4083 & 41. & $358,814.32$ & $2,473.95$ & 20 & 68 & $3,036.14$ & \multirow{3}{*}{$\begin{array}{c}\text { Pen } 60 / 70+\mathrm{Asb} \\
8 \%(6 \%)\end{array}$} \\
\hline & 0.0472 & 90.7475 & 2.2060 & 5.5512 & 0.8018 & 0.1 & -1.00000 & 6.5 & 6.046 & 65.545 & 17.535 & 11.493 & 8.4083 & 41 & $111,488.08$ & 768.69 & 35 & 95 & 509.26 & \\
\hline & -0.4322 & 110.9005 & 2.2060 & 5.5512 & 0.8018 & 0.1 & -1.00000 & 6.5 & 6.046 & 65.545 & 17.535 & 11.493 & 8.4083 & & $36,966.95$ & 254.88 & 50 & 122 & 279.83 & \\
\hline \multirow{3}{*}{$6.3 \mathrm{a}$} & 0.9836 & 568.1410 & 2.0546 & 5.5512 & 1.3000 & $\mathrm{l}$ & 0.00000 & 6.5 & 6.046 & 65.545 & 17.535 & 11.4 & 8.40 & & 82.14 & $6,639.57$ & 20 & 68 & $6,816.99$ & \multirow{3}{*}{$\begin{array}{c}\text { Pen } 6070+\text { Asb } \\
8 \%(6 \%)\end{array}$} \\
\hline & 0.4005 & 877.4760 & 2.0546 & 5.5512 & 1.3000 & 1 & 0.00000 & 6.5 & 6.046 & 65.545 & 17.535 & 11.493 & 8.4083 & 41. & $251,490.61$ & $1,733.98$ & 35 & 95 & $1,400.38$ & \\
\hline & -0.2351 & 1214.6813 & 2.0546 & 5.5512 & 1.3000 & $\mathrm{l}$ & 0.00000 & 6.5 & 6.046 & 65.545 & 17.535 & 11.493 & 8.4083 & 41. & $58,195.55$ & 401.25 & 50 & 122 & 563.47 & \\
\hline \multirow{3}{*}{$6.4 a$} & \begin{tabular}{|l|}
1.2612 \\
\end{tabular} & 4650.5437 & 1.9361 & 5.5512 & 1.7983 & 10 & 1.00000 & 6.5 & 6.046 & 65.545 & 17.535 & 11.493 & 8.4083 & 41. & $1,824,736.33$ & $12,581.19$ & 20 & 68 & $11,555.78$ & \multirow{3}{*}{$\begin{array}{c}\text { Pen } 6070+\text { Asb } \\
8 \%(6 \%)\end{array}$} \\
\hline & \begin{tabular}{|l|l|}
0.7048 \\
\end{tabular} & 8484.6914 & 1.9361 & 5.5512 & 1.7983 & 10 & 1.00000 & 6.5 & 6.046 & 65.545 & 17.535 & 11.493 & 8.4083 & 41. & $506,708.04$ & $3,493.65$ & 35 & 95 & $4,144.87$ & \\
\hline & 0.0053 & 13304.2729 & 1.9361 & 5.5512 & 1.7983 & 10 & 1.00000 & 6.5 & 6.046 & 65.545 & 17.535 & 11.493 & 8.4083 & 41. & 228.17 & 697.95 & 50 & 122 & $1,695.25$ & \\
\hline \multirow{3}{*}{ 7.1a } & -0.3014 & 9.3041 & 2.4855 & 6.6837 & 0.3035 & 0.01 & -2.00000 & 6.5 & 3.720 & 78.823 & 17.556 & 13.838 & 8.4083 & 41.3 & $49,953.07$ & 344.42 & 20 & 68 & 828.94 & \multirow{3}{*}{$\begin{array}{c}\text { Pen } 60 / 70+\text { Asb } \\
8 \%(7 \%)\end{array}$} \\
\hline & $\mid-0.5991$ & 10.2979 & 2.4855 & 6.6837 & 0.3035 & 0.01 & -2.00000 & 6.5 & 3.720 & 78.823 & 17.556 & 13.838 & 8.4083 & 41.3 & $25,170.39$ & 173.54 & 35 & 95 & 305.76 & \\
\hline & -0.8424 & 11.1102 & 2.4855 & 6.6837 & 0.3035 & 0.01 & -2.00000 & 6.5 & & 78.823 & 17.556 & & 8.4 & & & 99.11 & 50 & 122 & 194.00 & \\
\hline \multirow{3}{*}{$7.2 \mathrm{a}$} & 0.4751 & 76.1594 & 2.2868 & 6.6837 & 0.8018 & 0.1 & -1.00000 & 6.5 & 3.720 & 78.823 & 17.556 & 13.838 & 8.4083 & 41. & $298,613.88$ & $2,058.88$ & 20 & 68 & $2,312.30$ & \multirow{3}{*}{$\begin{array}{c}\text { Pen } 60 / 70+A s b \\
8 \%(7 \%)\end{array}$} \\
\hline & \begin{tabular}{|l|}
-0.0819 \\
\end{tabular} & 99.5748 & 2.2868 & 6.6837 & 0.8018 & 0.1 & -1.00000 & 6.5 & 3.720 & 78.823 & 17.556 & 13.838 & 8.4083 & 41. & $82,811.04$ & 570.97 & 35 & 95 & 703.30 & \\
\hline & -0.6080 & 121.6883 & 2.2868 & 6.6837 & 0.8018 & 0.1 & -1.00000 & 6.5 & 3.720 & 78.823 & 17.556 & 13.838 & 8.4083 & 41 & $24,662.59$ & 170.04 & 50 & 122 & 234.76 & \\
\hline & 0.9603 & 623.4065 & 2.1354 & 6.6837 & 1.3000 & 1 & 0.00000 & 6.5 & 3.720 & 78.823 & 17.556 & 13.8 & 8.4083 & & & $6,292.40$ & 20 & 68 & $5,386.53$ & \\
\hline $7.3 \mathrm{a}$ & 0.3205 & 962.8318 & 2.1354 & 6.6837 & 1.3000 & 1 & 0.00000 & 6.5 & 3.720 & 78.823 & 17.556 & 13.838 & 8.4083 & & $209,159.65$ & $1,442.11$ & 35 & 95 & $2,049.84$ & \\
\hline & -0.3770 & 1332.8385 & 2.1354 & 6.6837 & 1.3000 & 1 & 0.00000 & 6.5 & 3.720 & 78.823 & 17.556 & 13.838 & 8.4083 & 41.3 & $41,977.38$ & 289.43 & 50 & 122 & 396.03 & \\
\hline & \begin{tabular}{|l|l|}
1.2764 \\
\end{tabular} & 5102.9215 & 2.0170 & 6.6837 & 1.7983 & 10 & 1.00000 & 6.5 & 3.720 & 78.823 & 17.556 & 13.838 & 8.4083 & 41.3 & $1,889,727.45$ & $13,029.29$ & 20 & 68 & $9,702.57$ & \\
\hline 7.4a & \begin{tabular}{|l|}
0.6658 \\
\end{tabular} & 9310.0328 & 2.0170 & 6.6837 & 1.7983 & 10 & 1.00000 & 6.5 & 3.720 & 78.823 & 17.556 & 13.838 & 8.4083 & 41.3 & $463,264.87$ & $3,194.12$ & 35 & 95 & $5,052.02$ & \\
\hline & $\mid$\begin{tabular}{|c|}
-0.1017 \\
\end{tabular} & 14598.4351 & 2.0170 & 6.6837 & 1.7983 & 10 & 1.00000 & 6.5 & 3.720 & 78.823 & 17.556 & 13.838 & 8.4083 & 41.3 & $79,128.68$ & 545.58 & 50 & 122 & $1,102.26$ & \\
\hline
\end{tabular}




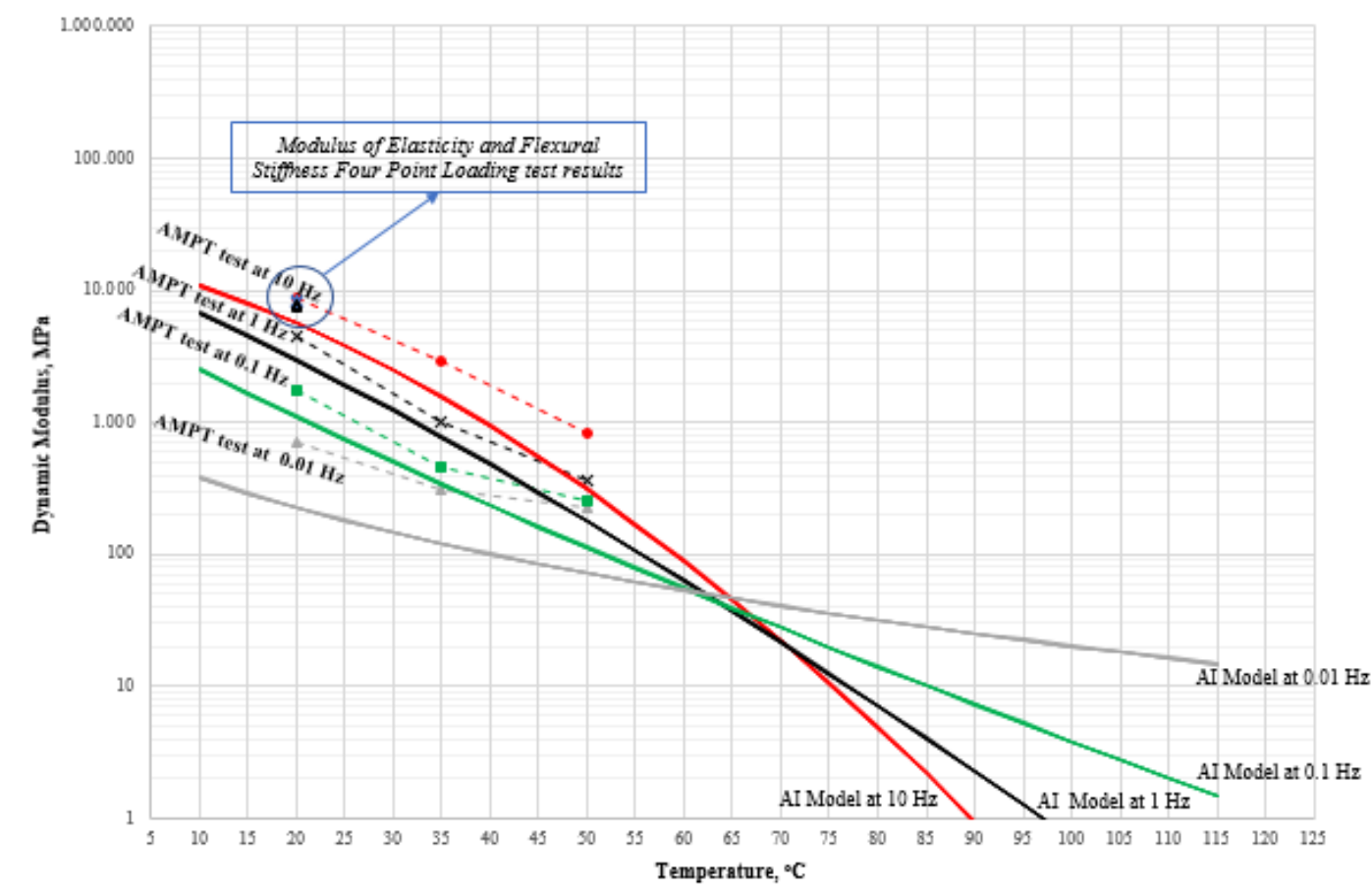

Fig. 2. The dynamic modulus relationship ( $\left.E^{*}\right)$ of AI model with modulus of elasticity and flexural stiffness from four point loading test result and AMPT testing of HMA without asbuton in asphalt content $6 \%$

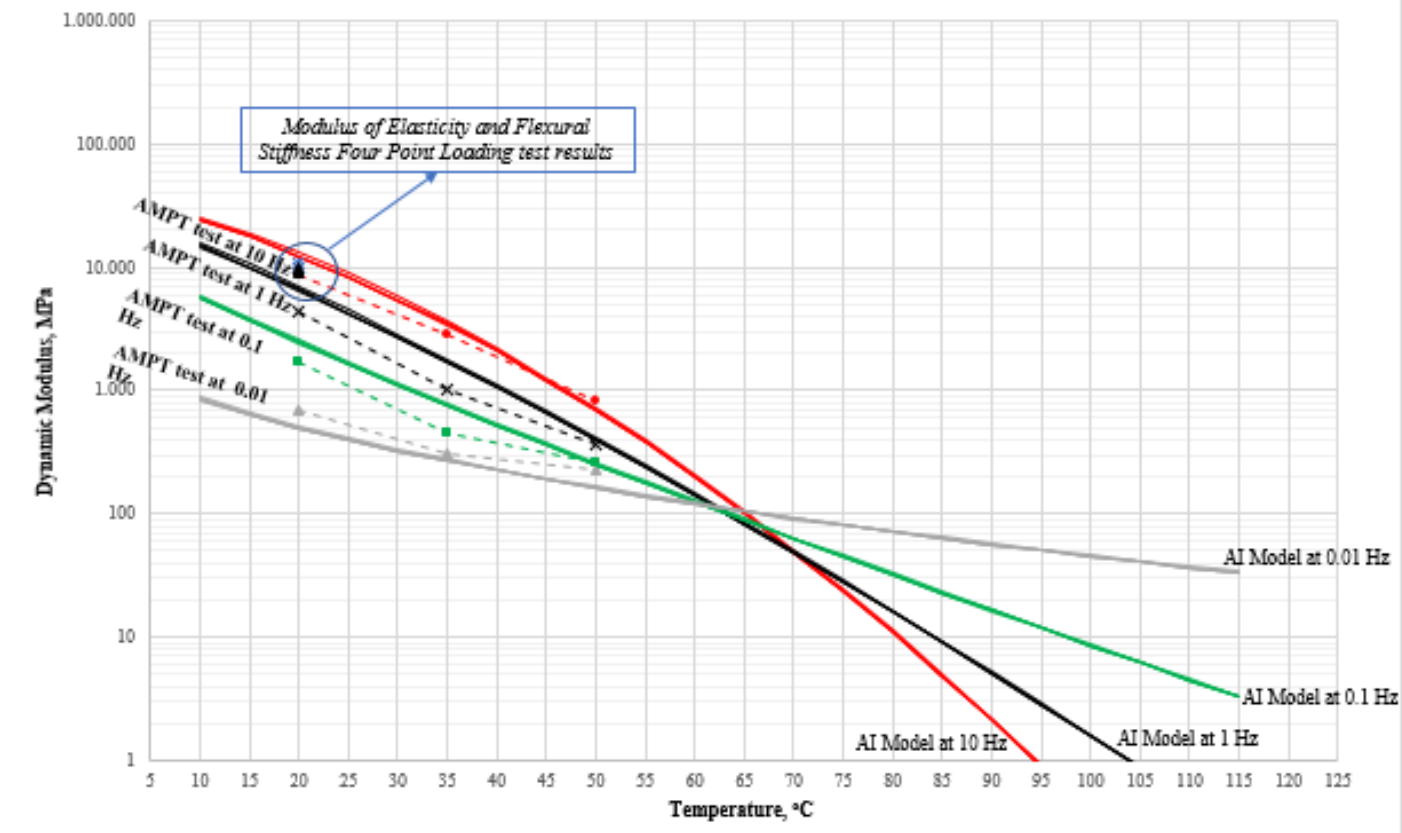

Fig. 3. The dynamic modulus relationship $(\mathrm{E} *$ ) of AI model with modulus of elasticity and flexural stiffness of four point loading test result and AMPT testing of HMA with $8 \%$ asbuton in asphalt content $6 \%$ 

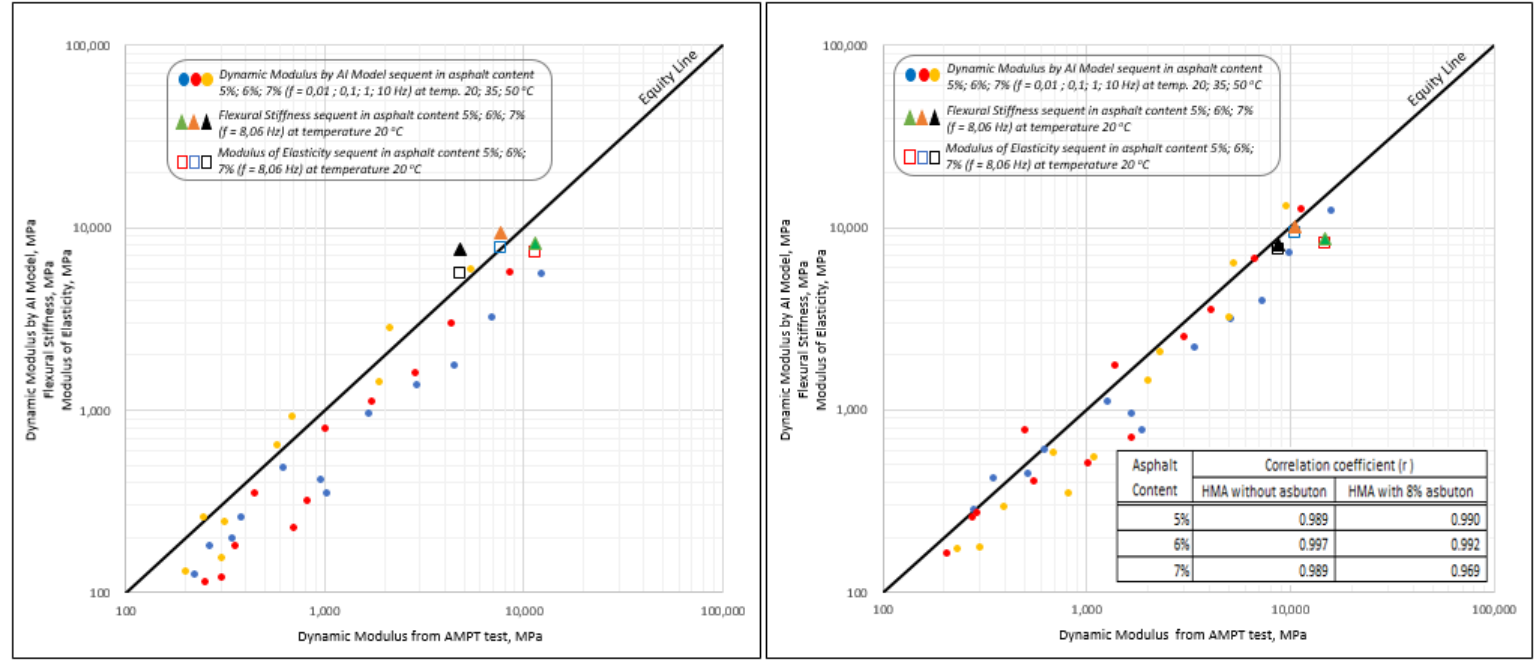

Fig. 4. The dynamic modulus relationship ( $\left.\mathrm{E}^{*}\right)$ of AI model with modulus of elasticity and flexural stiffness of Four Point Loading test result and AMPT testing of HMA without and with $8 \%$ asbuton

\subsection{Fatigue cracking}

The test of fatigue crack used is Four Point Loading. This test method refers to ASTM D7460-10 with a frequency of $8.06 \mathrm{~Hz}$ (equivalent to vehicle speed of $35.54 \mathrm{~km} / \mathrm{h}$ ) with sinusoidal loading at $20{ }^{\circ} \mathrm{C}$. The concept of constant stress testing using fatigue refers to the AI model, then test results and models are verified by statistical analysis with the steps shown in the Figure 5 .

The amount of stress is maintained by maintaining the load, the strain that occurs will be greater as the cycle increases. In this test the fatigue life is achieved when the flexural stiffness has been reduced by $50 \%$ of the initial value because at this point it is considered to be a shift of the crack, from microcrack to crack propagation / macrocrack (Rowe and Bouldin, 2000).

Summary of fatigue crack test results of HMA with $8 \%$ asbuton is presented in Table 3 and for the other mixtures showing the same behavior.

The result of fatigue crack test for asphalt mixture without and with $8 \%$ asbuton were then plotted into fatigue life correlation graph with flexural stiffness, shown in Figure 6 and 7 below this. As for the other asphalt mixture shows the same behavior.

Table 3. Fatigue $\left(\mathrm{N}_{\mathrm{f}}\right)$ test results of HMA with $8 \%$ asbuton

\begin{tabular}{|c|c|c|c|c|c|c|c|c|c|c|}
\hline \multirow{2}{*}{$\begin{array}{l}\text { Type of } \\
\text { HMA }\end{array}$} & \multirow{2}{*}{$\begin{array}{l}\text { Asp } \\
\text { halt } \\
\text { Cont } \\
\text { ent } \\
(\%) \\
\end{array}$} & \multirow{2}{*}{$\begin{array}{l}\text { Initial } \\
\text { Stress } \\
(\mathrm{kPa})\end{array}$} & \multirow{2}{*}{$\begin{array}{c}\text { Initial } \\
\text { Strain } \\
\mu \varepsilon\end{array}$} & \multicolumn{3}{|c|}{$\begin{array}{l}\text { Flexural Stiffness } \\
(\mathrm{MPa})\end{array}$} & \multicolumn{2}{|c|}{$\begin{array}{c}\text { Modulus of } \\
\text { Elasticity (MPa) }\end{array}$} & \multirow{2}{*}{$\begin{array}{c}\mathrm{N}_{\mathrm{f}} \\
\text { (Cycles) }\end{array}$} & \multirow[b]{2}{*}{ Explanation } \\
\hline & & & & Initial & Current & $\begin{array}{l}\text { Termi } \\
\text { nation }\end{array}$ & Initial & $\begin{array}{c}\text { Curre } \\
\text { nt }\end{array}$ & & \\
\hline \multirow{12}{*}{$\begin{array}{c}\text { Pen } \\
60 / 70+ \\
\text { Asb 8\% }\end{array}$} & \multirow{3}{*}{5} & 2,065 & 227 & 9,093 & 4,538 & 4,547 & 9,661 & 4,821 & 102,780 & \\
\hline & & 2,455 & 296 & 8,255 & 4,056 & 4,128 & 8,783 & $4, .315$ & 20,550 & \\
\hline & & 2,656 & 366 & 7,257 & 3,449 & 3,629 & 7,717 & 3,667 & 12,150 & \\
\hline & \multirow{6}{*}{6} & 2,064 & 221 & 9,351 & 7,686 & 4,675 & 9,953 & 8,181 & 312,600 & discontinued \\
\hline & & & & & & & & & $1,111,700$ & $\begin{array}{c}\text { Extrapolation } \\
\text { result }\end{array}$ \\
\hline & & 2,453 & 248 & 9,883 & 7,945 & 4,941 & $\begin{array}{c}10,51 \\
5\end{array}$ & 8,453 & 175,110 & discontinued \\
\hline & & & & & & & & & 669,510 & $\begin{array}{c}\text { Extrapolation } \\
\text { result }\end{array}$ \\
\hline & & 2,649 & 292 & 9,076 & 7,332 & 4,538 & 9,654 & 7,798 & 124,920 & discontinued \\
\hline & & & & & & & & & 360,200 & $\begin{array}{c}\text { Extrapolation } \\
\text { result }\end{array}$ \\
\hline & \multirow{3}{*}{7} & 2,065 & 272 & 7,594 & 3,780 & 3,787 & 8,088 & 4,026 & 274,320 & \\
\hline & & 2,445 & 319 & 7,668 & 3,817 & 3,834 & 8,164 & 4,064 & 157,790 & \\
\hline & & 2,656 & 358 & 7,412 & 3,684 & 3,706 & 7,880 & 3,917 & 93,780 & \\
\hline
\end{tabular}

note: * discontinued, because if testing continues it will take a very long time, for it must be extrapolated 


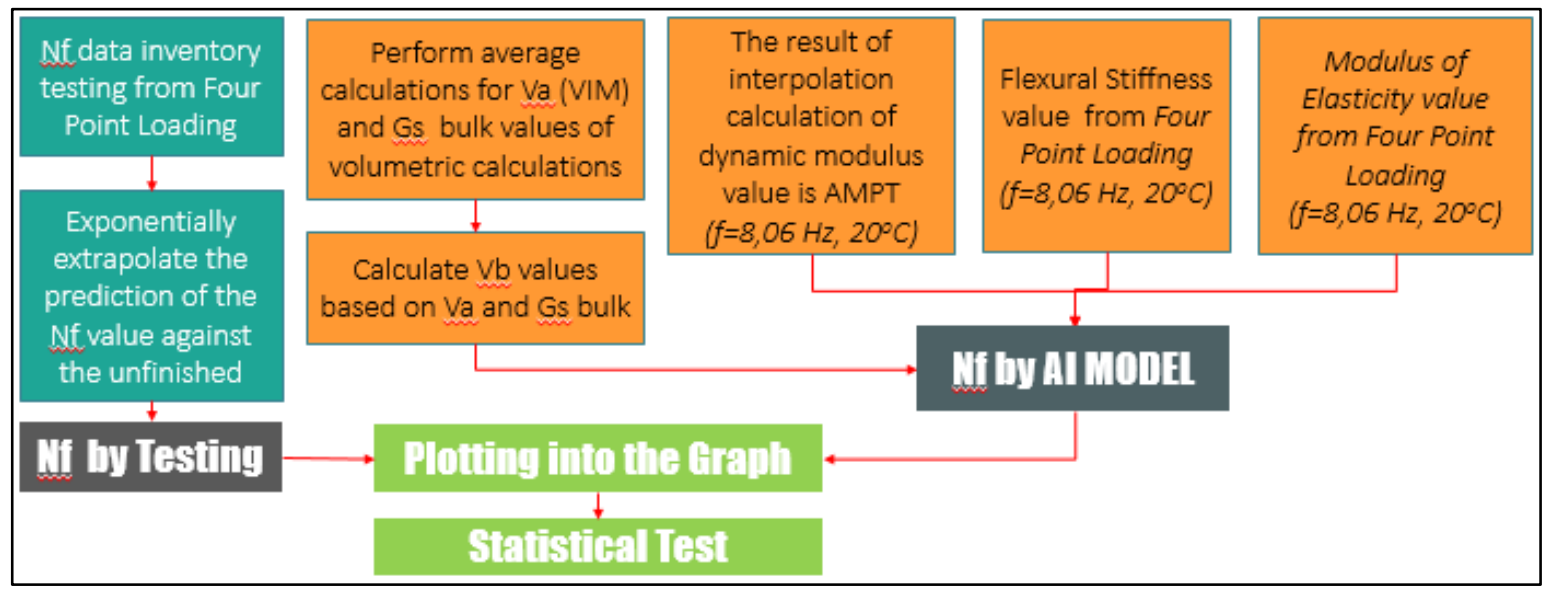

Fig. 5. The steps to verify the Four Point Loading test results on the fatigue life $\left(\mathrm{N}_{\mathrm{f}}\right)$ AI model

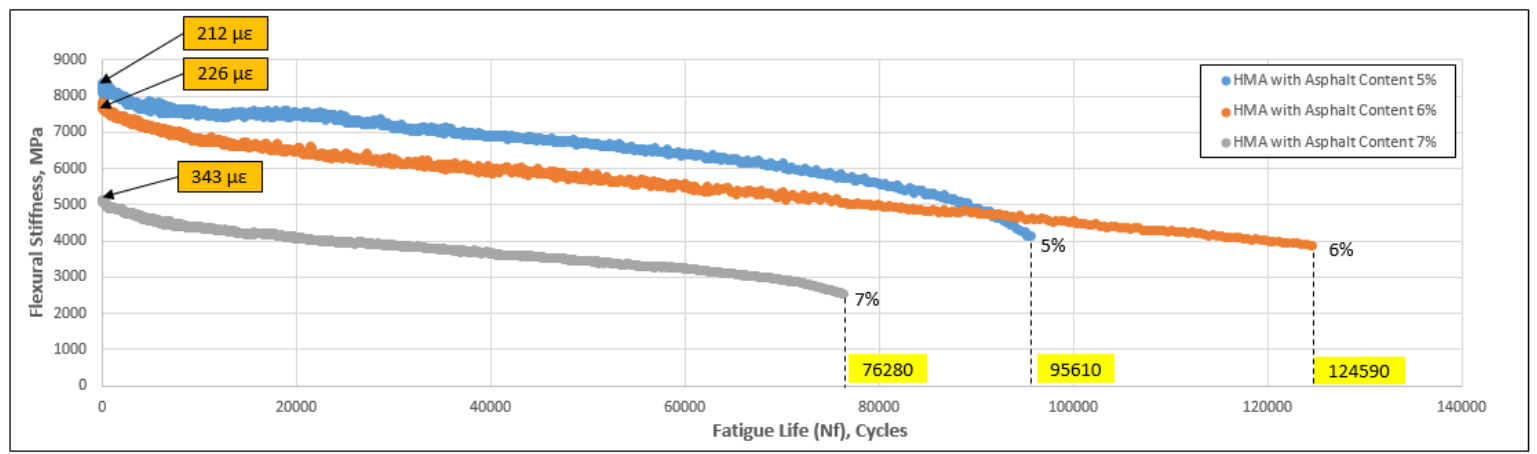

Fig. 6. Relationship of flexural stiffness with fatigue life $\left(\mathrm{N}_{\mathrm{f}}\right)$ for HMA without asbuton at a stress of $1750 \mathrm{kPa}$

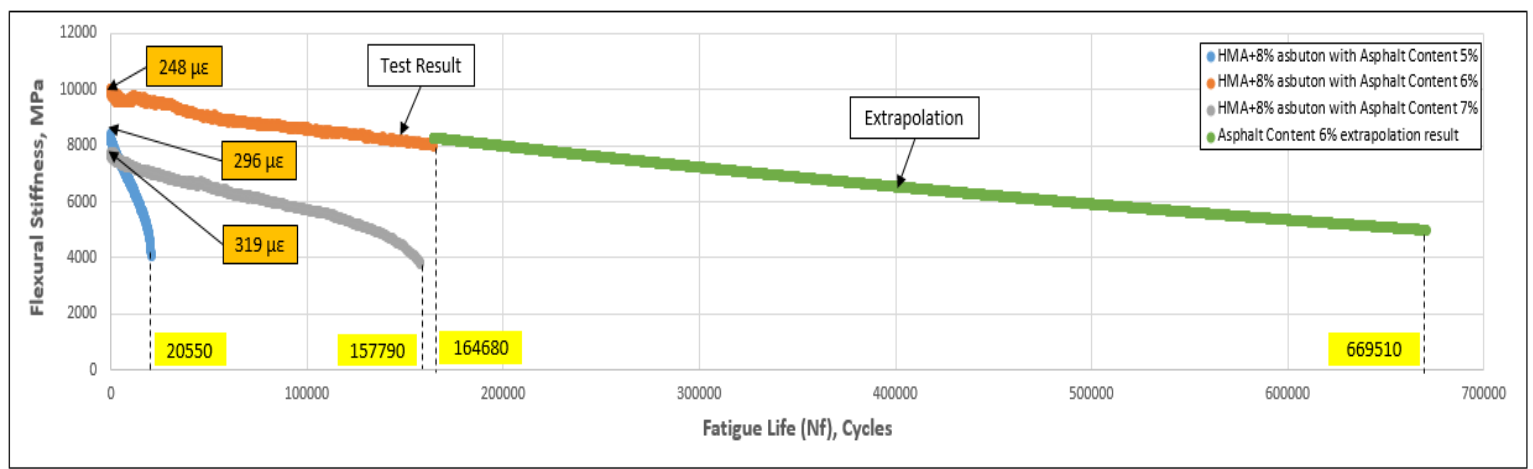

Fig. 7. Test results and extrapolation flexural stiffness vs fatigue life (Nf) for HMA with $8 \%$ asbuton at a stress of $2450 \mathrm{kPa}$

In this study, there were 3 (three) tests of unfinished fatigue life, all three for asphalt mixture $8 \%$ asbuton with $6 \%$ asphalt content. The tests were carried out at a stress of $2065 \mathrm{kPa}, 2450 \mathrm{kPa}$ and $2650 \mathrm{kPa}$ with fatigue life when discontinued respectively were 312,600 cycles, 175,110 cycles and 124,920 cycles which can be seen in Table 3.

The predicted fatigue life can be performed by extrapolating the graph of the decrease in the flexural rigidity modulus to fatigue life to $50 \%$ of the initial flexural stiffness modulus (NCHRP, 2010).

If the fatigue life $\left(\mathrm{N}_{\mathrm{f}}\right)$ values have been calculated based on the AI model and the Four Point Loading test results are plotted into the graph, then the AI model calculation results obtained and the test results show that it is generally adjacent.

The relationship between test results using Four Point Loading and model, can be seen in Figure 8. Generally, from the results of the fatigue crack testing using Four Point Loading, it has been shown that asphalt mixture with $8 \%$ asbuton has a fatigue life $\left(\mathrm{N}_{\mathrm{f}}\right)$ greater than asphalt mixture without asbuton. This illustrates that the asphalt mixture with $8 \%$ asbuton is more resistant to fatigue than the asphalt mixture without asbuton.

Verification based on the results of statistical analysis of the fatigue life using the four points loading test results 
with AI Model is good enough in predict fatigue life, with 95\% confidence level and significance (error) 5\%.

The relationship between the test results and the fatigue crack model is shown in Figures 9. From the analysis showed that the mixture was asphalt without and with $8 \%$ asbuton, most of fatigue life $\left(\mathrm{N}_{\mathrm{f}}\right)$ from Four Point
Loading test were lower than model. The laboratory model need to be calibrated for variations wheel track and loading time occuring on site.

Table 4. Result of calculation of fatigue life (Nf) model AI based on dynamic modulus value (E*) from AMPT test interpolated at frequency of $8.06 \mathrm{~Hz}$ and temperature $20^{\circ} \mathrm{C}$

\begin{tabular}{|c|c|c|c|c|c|c|c|c|}
\hline \multirow[t]{2}{*}{ Type HMA } & \multirow[t]{2}{*}{$\begin{array}{c}\text { Strain } \\
(\mu \varepsilon)\end{array}$} & $\begin{array}{c}\text { Dynamic } \\
\text { Modulus } \\
\text { AMPT (MPa) } \\
\end{array}$ & \multirow{2}{*}{$\begin{array}{c}\text { Dynamic } \\
\text { Modulus } \\
\text { AMPT (Psi) } \\
\text { (x 145) }\end{array}$} & \multirow[t]{2}{*}{$\begin{array}{l}\mathrm{Va} \\
(\%)\end{array}$} & \multirow[t]{2}{*}{$\begin{array}{l}\mathrm{Vb} \\
(\%)\end{array}$} & \multirow[t]{2}{*}{ M } & \multirow[t]{2}{*}{$\begin{array}{l}\text { Nf AI Model } \\
\text { (cycles) }\end{array}$} & \multirow{2}{*}{$\begin{array}{c}\text { Nf from } \\
\text { Four Point } \\
\text { Loading } \\
\text { Testing } \\
\text { (cycles) } \\
\end{array}$} \\
\hline & & $20{ }^{\circ} \mathrm{C} ; 8.06 \mathrm{~Hz}$ & & & & & & \\
\hline \multirow{3}{*}{$\begin{array}{c}\text { Pen } 60 / 70 \\
(5 \%)\end{array}$} & 212 & $11,327.17$ & $1,642,439.17$ & 8.32 & 10.84 & -0.60 & $120,617.11$ & $95,610.00$ \\
\hline & 283 & $11,327.17$ & $1,642,439.17$ & 8.32 & 10.84 & -0.60 & $46,617.73$ & $22,700.00$ \\
\hline & 374 & $11,327.17$ & $1,642,439.17$ & 8.32 & 10.84 & -0.60 & $18,623.44$ & $13,010.00$ \\
\hline \multirow{3}{*}{$\begin{array}{c}\text { Pen } 60 / 70 \\
(6 \%)\end{array}$} & 226 & $7,7,698.22$ & $1,116,241.21$ & 5.84 & 13.17 & 0.01 & $\begin{array}{c}558,008.68 \\
\end{array}$ & $124,590.00$ \\
\hline & 263 & $7,698.22$ & $1,116,241.21$ & 5.84 & 13.17 & 0.01 & $338,795.37$ & $117,280.00$ \\
\hline & 314 & $7,698.22$ & $1,116,241$ & 5.84 & 13.17 & 0.01 & $189,067.27$ & $58,220.00$ \\
\hline \multirow{3}{*}{$\begin{array}{c}\text { Pen } 60 / 70 \\
(7 \%)\end{array}$} & 343 & $4,745.24$ & $6888,059.82$ & 3.61 & 15.51 & 0.59 & $800,285.83$ & $76,280.00$ \\
\hline & 307 & $4,745.24$ & $688,059.82$ & 3.61 & 15.51 & 0.59 & $1,152,724.92$ & $94,350.00$ \\
\hline & 461 & $4,745.24$ & $688,059.82$ & 3.61 & 15.51 & 0.59 & $302,453.30$ & $23,380.00$ \\
\hline \multirow{3}{*}{$\begin{array}{c}\text { Pen } \\
60 / 70+8 \% \\
\text { asbuton }(5 \%) \\
\end{array}$} & 227 & $14,840.56$ & $2,151,880.77$ & 8.65 & 10.78 & -0.65 & $67,602.00$ & $102,780.00$ \\
\hline & 296 & $14,840.56$ & $2,151,880.77$ & 8.65 & 10.78 & -0.65 & $28,224.07$ & $20,550.00$ \\
\hline & 366 & $14,840.56$ & $2,151,880.77$ & 8.65 & 10.78 & -0.65 & $14,035.39$ & $12,150.00$ \\
\hline \multirow{3}{*}{$\begin{array}{c}\text { Pen } \\
60 / 70+8 \% \\
\text { asbuton }(6 \%) \\
\end{array}$} & 221 & $10,534.31$ & $1,527,474.71$ & 6.05 & 13.11 & $\begin{array}{l}-0.03 \\
\end{array}$ & (419,568.29 & $312,840.00$ \\
\hline & 248 & $10,534.31$ & $1,527,474.71$ & 6.05 & 13.11 & -0.03 & $287,115.84$ & $175,110.00$ \\
\hline & 292 & $10,534.31$ & $1,527,474.71$ & 6.05 & 13.11 & -0.03 & $167,734.60$ & $124,920.00$ \\
\hline \multirow{3}{*}{$\begin{array}{c}\text { Pen } \\
60 / 70+8 \% \\
\text { asbuton }(7 \%)\end{array}$} & 272 & $8,772.22$ & $1,271,971.97$ & 3.72 & 15.46 & 0.56 & 960,500.99 & $274,320.00$ \\
\hline & 319 & $8,772.22$ & $1,271,971.97$ & 3.72 & 15.46 & 0.56 & $569,445.64$ & $157,790.00$ \\
\hline & 358 & $8,772.22$ & $1,271,971.97$ & 3.72 & 15.46 & 0.56 & $388,897.40$ & $93,780.00$ \\
\hline
\end{tabular}
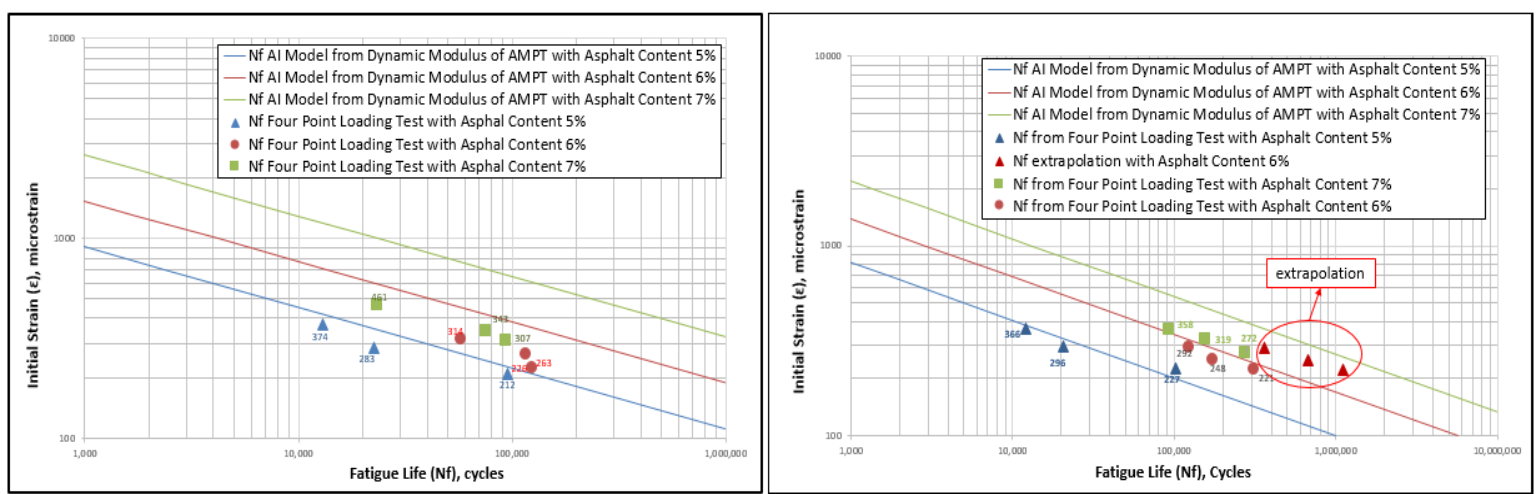

Fig. 8. Fatigue life $\left(\mathrm{N}_{\mathrm{f}}\right)$ relationship of HMA from four point loading test results and $\mathrm{N}_{\mathrm{f}} \mathrm{AI}$ model of dynamic modulus (E*) AMPT interpolated for HMA without and with $8 \%$ asbuton at frequencies $8.06 \mathrm{~Hz}$ and temperature $20{ }^{\circ} \mathrm{C}$ 


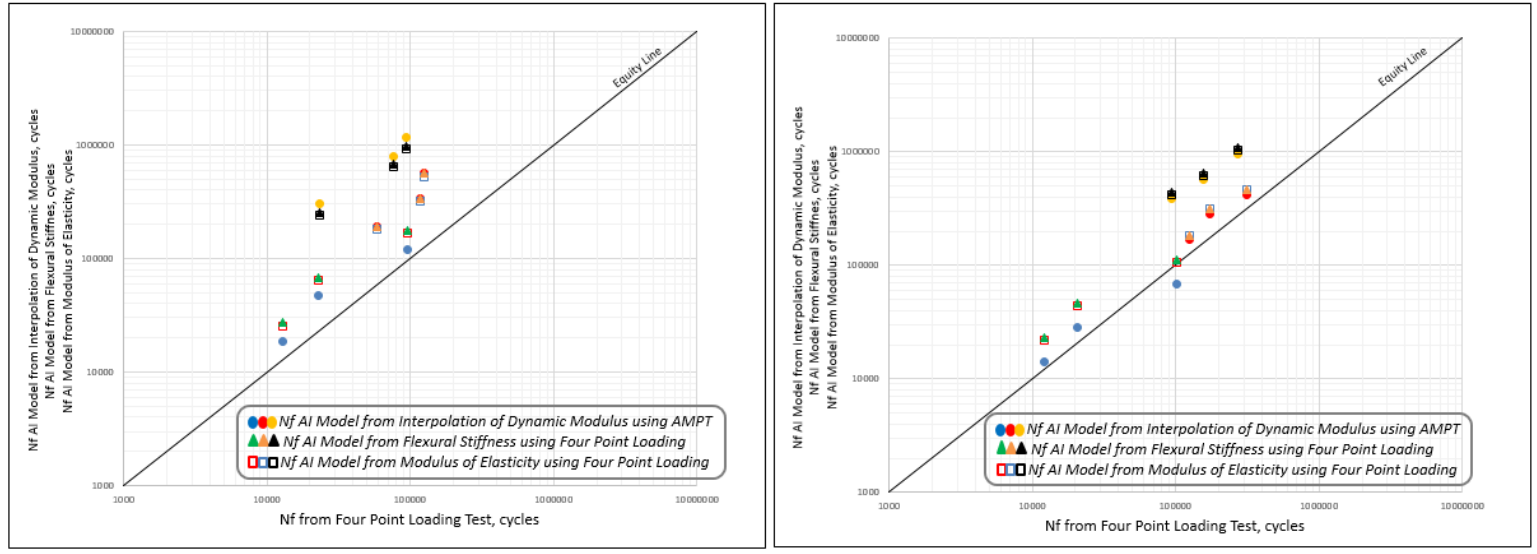

Fig. 9. Fatigue life $\left(\mathrm{N}_{\mathrm{f}}\right)$ relationship of four points loading test with $\mathrm{AI}$ fatigue crack model based on dynamic modulus $\left(E^{*}\right)$ of AMPT and flexural stiffness and modulus of elasticity from four points loading for HMA without and with $8 \%$ asbuton at frequency $8.06 \mathrm{~Hz}$ at $20^{\circ} \mathrm{C}$

\section{Conclusions}

From data analysis explained above some conclusions can be drawn, as follows:

a. The result of verification of relation between dynamic modulus (E*) using AMPT testing with dynamic modulus model by Asphalt Institute, showed fairly consistent result indicated by correlation coefficient (r) which ranged from 0.969 to 0.997 .

Yet, the test results show that most of the modulus values are greater than those computed by the model. This, if seen from its influence on flexible pavement design, that the model is the safe side.

b. The result of verification between fatigue crack/fatigue life $\left(\mathrm{N}_{\mathrm{f}}\right)$ Four Point Loading with AI Model is good enough in predict fatigue life, with 95\% confidence level and significance (error) 5\%.

From the analysis showed that the mixture was asphalt without and with $8 \%$ asbuton, most of fatigue life $\left(\mathrm{N}_{\mathrm{f}}\right)$ using Four Point Loading were lower than model. The laboratory model need to be calibrated for variations wheel tracks and loading time occuring on site. The calibration factor of this model is still to be subject for futher research.

\section{References}

1. N. Suaryana, Performance Evaluation of Asphlat Stone Matrix Using Grain Asbuton as a Stabilizer and Filler, Dissertation, Civil Engineering Doctoral Study Program, Institut Teknologi Bandung (2014)

2. E.J Yoder and M.W Witczak, Principles of Pavement Design, Second Edition, USA: John Wiley \& Sons, Inc (1975)

3. Asphalt Institute, Principles of Construction of Hot Mix Asphalt Pavement, The Asphalt Institute (1982)

4. J. Yu and G. Zou., Asphalt Pavement Fatigue Cracking Prediction Model with Mode Factor, International Journal of Pavement Research and Technology, Vol. 6 No.2, 123-129 (2013)
5. G.M Rowe and M.G Bouldin, Improved Techniques to Evaluate The Fatigue Resistance of Asphaltic Mixtures, Proceedings of 2nd Euroasphalt and Eurobitumen Congress, Barcelona, Spain (2000)

6. Y.H Huang, Pavement Analysis and Design Second Edition, New Jersey: Prentice-Hall Inc (2012)

7. NCHRP, Validating the Fatigue Endurance Limit for Hot Mix Asphalt, Report 646, Transportation Research Board of National Academies, Washington DC (2010) 